\title{
Dosimetric Importance of the Implementation of Daily Image Guidance in Radiotherapy Practice
}

\author{
Hridya V T ${ }^{1,2 *}$, D Khanna ${ }^{1}$, Aswathi Raj ${ }^{1,2}$, Sathish Padmanabhan ${ }^{2}$, P Mohandass ${ }^{3}$
}

\begin{abstract}
Background: The purpose of this study is to show the dosimetric importance of using daily image guidance in radiotherapy treatment. Methods: A sample of 30 patients with various head and neck cancers were retrospectively selected for the studies. The prescribed dose, $66 \mathrm{~Gy} / 33$ fractions was used for all patients who received 7 to 9 beams, 6 MV Intensity Modulated Radiation Therapy (IMRT) plan delivered by Varian Truebeam STx. Before the first fraction of the treatment, the patient's shift corresponds to isocenter was noted and corrected. Subsequently, the images were taken daily for all 30 patients and the maximum, mode, median and mean of all shifts were recorded and applied to the base plan and recalculated for quantitative analysis of tumour coverage and Organ at Risk's (OARs) doses using various dosimetric parameters such as Homogeneity Index (HI), Conformation Number (CN), Conformity Index (CI), Coverage Index (COVI), Dose Gradient Index (DGI) and Unified Dosimetry Index (UDI) of shift plan. Results: The results showed the Planning Target Volume (PTV) and the OARs values deteriorated from its base plan values in the various shift plans created by applying the patient setup errors analyzed using image guidance. Mean dose of maximum shift plan with a significant $P$ value of 0.002 , D2 $\%$ of maximum shift plan with a significant $P$ value 0.028 , the D98\% Values of maximum and mode plans with the significant $\mathrm{P}$ value 0.004 each, the $\mathrm{D} 50 \%$ and $\mathrm{D} 80 \%$ values of the maximum shift plans with their significant $\mathrm{P}$ values 0.001 and 0.002 respectively. Also, $\mathrm{HI} 95 \%$, CN95\% and COVI values of the maximum shift plan showed much variation with significant $P$ values of $0.004,0.040$ and 0.0004 respectively from their base plan values. There were significant changes observed in OARs values between base plan and shift plans. Conclusion: Implementation of daily image guidance in radiotherapy is mandatory taking into account of its dosimetric importance and to achieve the goal of radiotherapy practice of sparing the critical organs without compromising the target coverage.
\end{abstract}

Keywords: Head and Neck cancers- IGRT imaging- shift in IGRT and Imaging in Radiotherapy

Asian Pac J Cancer Prev, 23 (1), 355-361

\section{Introduction}

The main aim of radiotherapy is to relieve the cancer cells from their multiplication potential which itself makes it an important factor of cancer treatment (Baskar et al., 2012). And it is very significant in radiotherapy to maximize radiation exposure and dose deposition in the cancer cells and at the same time, to reduce the radiation exposure over the adjacent normal cells to its minimum (Van't et al., 1997). Radiotherapy is a technology-oriented treatment modality and it has evolved into an extremely complex technique from the conventional treatment modes to highly advanced conformal radiotherapy techniques (Garibaldi et al., 2017). Advancements in radiotherapy have occurred in all sectors of radiotherapy workflow, starting from the different imaging modalities like Computed Tomography [CT] simulation, Magnetic Resonance Imaging [MRI] or Positron Emission Tomography [PET] for the accurate identification and delineation of target volumes (PTV) and OARs (Metcalfe et al., 2013; Ashamalla et al., 2005), then with the advanced registration and fusion algorithms in Treatment Planning Systems (TPS) to aid in precise contouring work as well as the highly sophisticated and advanced radiobiology-oriented treatment planning optimization tools (Nahun et al., 2012). In the treatment execution process great strides have been made by the emergence of new image-guided techniques with which the tumor can be localized very accurately before and during the treatment procedure (Jaffray et al., 2012; Nabavizadeh et al., 2016).

Intensity-modulated radiotherapy (IMRT) is a radiotherapy technique that allows the delivery of steep dose gradients with multiple beam angles to modulate the intensity of the dose. This technique helps in delivering a highly conformal dose to the tumour along with the advantage of sparing the adjacent OARs to a higher extent 
as compared to the old techniques used (Cheung et al., 2006). IMRT is the most suitable treatment modality compared to 3 Dimensional Conformal Radiotherapy (3DCRT) for heterogeneous head and neck cancers because these techniques take into account of the complex shape of the target and the close proximal OARs. The IMRT technique addresses the discrepancies like under dosage of target volumes and overexposure of OARs as well as giving better dose hotspot, improved target volume dose distribution and reduced radiation dose to OARs (Hummel et al., 2010; Murshad et al., 2004).

Image-guided radiotherapy [IGRT] is a step ahead of IMRT and it has played an important role throughout radiotherapy history as its introduction and subsequent innovations have revolutionized cancer treatment. The IGRT uses advanced imaging modalities for accounting and thereby adjusting the target motion uncertainties and positional uncertainties (Kearney et al., 2020).

The need for image guidance in radiotherapy practice is very important due to many reasons like tumor regression with treatment response, weight loss due to less nutrition or perhaps growth itself inspired by treatment. There could be interfractional changes in treatment between each radiation fraction due to variations in patient positioning. The patient setup in each radiotherapy fraction is affected by different factors such as variations in patient setup, dosimetric uncertainties, mechanical uncertainties and human errors (Weygand et al., 2016). There are many reports that have studied the impact of these errors on PTV coverage and OARs. A positional error can increase dosimetric error and it leads to the reduction of therapeutic index. Recent studies have examined how the setup errors in head and neck cancer patients treated with IMRT have influenced the customized immobilization systems and other patient factors (Contesini et al., 2017). A study which was carried out on the effect of setup errors and organ motion on prostate cancer treated with IMRT showed that in most of the recalculated plans, the rectal wall dose values were very much altered from the planned one despite the small variations seen in the Clinical Target Volume (CTV) coverage (Landoni et al., 2006).

The IGRT uses 2D or 3D images which allow for correcting the patient position to radiotherapy treatment position before radiation delivery and paves way for precise treatment delivery. The radiotherapy treatment has advanced from the early conventional methods of skin markings on the patient body to recent advancements in IGRT such as CT, Cone Beam CT (CBCT), MV and KV On-Board Imaging (OBI) that helps in precise internal anatomy based patient positioning during treatment. It is also significant to manage the imaging dose exposure to the patients as it may result in excessive dose to the OARs and increase the chance of inducing secondary cancers (Ding et al., 2018).

The practice of radiotherapy in hospitals is very much related to the workload or work quality in the respective department. Hence this present study is intended to find out the dosimetric effect on non-IGRT practices. The main aim of this study is to find out the level of dosimetric error that arises from the actual plan i.e. whether the approved IMRT delivery plan delivers the same intensity- modulated beam plan at the treatment position by the image guidance treatment procedures. Moreover in this study, the possible degrees of positional shift errors and its impact on the plan execution results has been verified by taking the maximum, mode, median and mean of the daily shift values.

\section{Materials and Methods}

\section{Patient Selection}

Thirty patients with various head and neck cancers were retrospectively selected for this study. The patients selected were in the age group of 45 to 75 years after getting approval from the institutional ethical committee.

\section{Treatment Planning}

The patients selected for the study were simulated in the headfirst supine position using a thermoplastic mould and shoulder retraction, for which $3 \mathrm{~mm}$ slice thickness CT images were acquired. The acquired images were then transferred to the Eclipse TPS version 15.06. The MRI images taken in the same position was sent to the TPS which was later registered and fused with the CT images for better localization of tumour and OARs and based on the treatment protocol, the target volumes, as well as the critical organs, were delineated. Initially, the Gross Tumour Volume GTV) was delineated involving all positive lymph nodes with the aid of fused images. Following the GTV, the Clinical Tumour Volume [CTV] was contoured taking into account of the primary tumour size, its involved nodes and then a margin for the microscopic spread. Planning Target Volume [PTV] was contoured by the expansion of the CTV by $5 \mathrm{~mm}$ in all directions [International Commission on Radiation Units and Measurements (ICRU) 50 and 62]. Then the critical organs or the OARs such as spinal cord, left parotid, right parotid, larynx, oral cavity, left eye, right eye, left optic nerve, right optic nerve, brainstem, mandible, left cochlea, right cochlea and optic chiasm were contoured. An additional healthy tissue was also defined which is the patient CT volume excluding the PTV volume.

The dose prescribed for the tumour volume of these patients taken for this study was 66Gy in 33 fractions (2Gy per fraction). For all the patients 7-9 beam IMRT plan was generated with gantry angles $0^{\circ}, 50^{\circ}, 100^{\circ}$, $150^{\circ}, 200^{\circ}, 250^{\circ}, 300^{\circ}$. The plan was optimized for $6 \mathrm{MV}$ X-ray photon delivered by Varian Truebeam STx linear accelerator equipped with an HD Multi-Leaf Collimator (MLC) with 120 leaves using Photon Optimizer (15.6.05) algorithm to achieve the given planning objectives. Dose calculation was done using the Anisotropic Analytical Algorithm (AAA) at $2.5 \mathrm{~mm}$ calculation grid size. The plan objectives for PTV were that at least $95 \%$ of the PTV should cover $95 \%$ of the dose prescribed and the dose maximum should not exceed a value of $107 \%$. The dose constraints followed for OARs like spinal cord, bilateral parotids, larynx, optic nerve, mandible, cochlea, brainstem, eye were D1\%<46 Gy, Dmean < 26 Gy; V30Gy $<45 \%$, Dmean $<45$ Gy, D1\% < 50Gy, D1\% < 61Gy, Dmean $<15$ Gy, D1\% < 54Gy, Dmax $<45$ Gy respectively where D $1 \%$ - Dose to $1 \%$ of volume, Dmean-Mean dose, 
V30Gy - Volume receiving 30 Gy and Dmax - Maximum dose respectively.

The plans were analysed and approved based on Dose Volume Histograms (DVH), tumour coverage and OAR dose. Before delivering the approved plan (base plan), a set of verification images were taken to ensure that the patient position is the same as the simulated position which was used for planning. The observed shifts in the patient position by matching methods was taken and recorded and as Mohandass et al., (2020) stated in his study any of the matching methods can be used during CBCT to check patient translation errors for the delivery of the VMAT head and neck patients. After verifying the position and making necessary corrections, the plan was delivered. The maximum, mode, median and mean of all observed shifts were calculated. Then the shift values were intentionally applied to the approved base plan, recalculated and was named as shift plans, to assess the dosimetric deviation from the original approved plan if the image verification procedure was not carried out continuously. The DVH and tumour coverage of these shift plans were also found and recorded. Various parameters such as Homogeneity Index (HI), Conformation Number $(\mathrm{CN})$ and Dose Gradient index (DGI) were used for evaluating the shift plan as compared to base plan. The homogeneity index is defined as;

$$
\text { Homogeneity Index }(H I)=\left(D_{2 \%}-D_{98 \%}\right) / D 50_{\%}
$$

Where D2\%, D98\% and D50\% are dose received by $2 \%, 98 \%$ and $50 \%$ volumes respectively. The ideal value of $\mathrm{HI}$ is 0 . The $\mathrm{CN}$ was calculated for each plan to evaluate the conformity of dose to target by the following formula;

$$
\mathrm{CN}_{95 \%}=(\mathrm{TVpi} / \mathrm{TV}) \times[\mathrm{TVpi} / \mathrm{Vpi}]
$$

Where TVpi is Target Volume within the 95\% prescribed isodose volume, $\mathrm{Vpi}$ is the Volume of $95 \%$ of prescribed isodose volume and TV is the tumour volume. The ideal values of $\mathrm{CN}$ should be 1 . The DGI was noted down using the formula PI/D50\% whose ideal value is 1 , where PI is the prescribed isodose volume and $\mathrm{D} 50 \%$ is the volume of $50 \%$ of prescribed isodose volume. The coverage index (COVI) as TVpi/ TV and Conformity Index (CI) as PI/TV were calculated and noted down whose ideal value is 1 . Unified Dosimetry Index (UDI) is tool used to compare and evaluate any given treatment plans by its deviations in terms of dose coverage, conformity, homogeneity, and dose gradient. It is calculated using the formula:

$$
U D I=U D I[C I] \times U D I[C F] \times U D I[H I] \times U D I[D G]
$$

whose ideal value is 1 , where the CI-coverage index, CF-conformity index, HI -Homogeneity Index and DG-gradient index. The mathematical logic-based UDI formula is:

$$
U D I=\left(\prod_{k=1}^{4} W_{k}\left[\left|1.0-D I_{k}\right|+0.1\right]\right) \times 10^{4}
$$

Where $\mathrm{D} I_{\mathrm{k}}$ is dosimetry index, each of the four indices and $\mathrm{Wk}$ is the weighting factors as per the relative importance of all the four components. For the OARs, the mean dose, the maximum dose and appropriate values of volume receiving $x \mathrm{~Gy}$ were noted. The calculated difference of the patients were analysed statistically using one sample ' $t$ ' test and considered significant if the $P$ value was less than 0.05 .

\section{Results}

The dose distribution of base plan and all other compared plans of Maximum, Mode, Median and Mean shift plans are shown in Figure 1.

Similar observations were plotted in other graphs generated. And the results show that the base plan parameters got deteriorated from their actual base plan values in the shift plans created by applying the maximum,

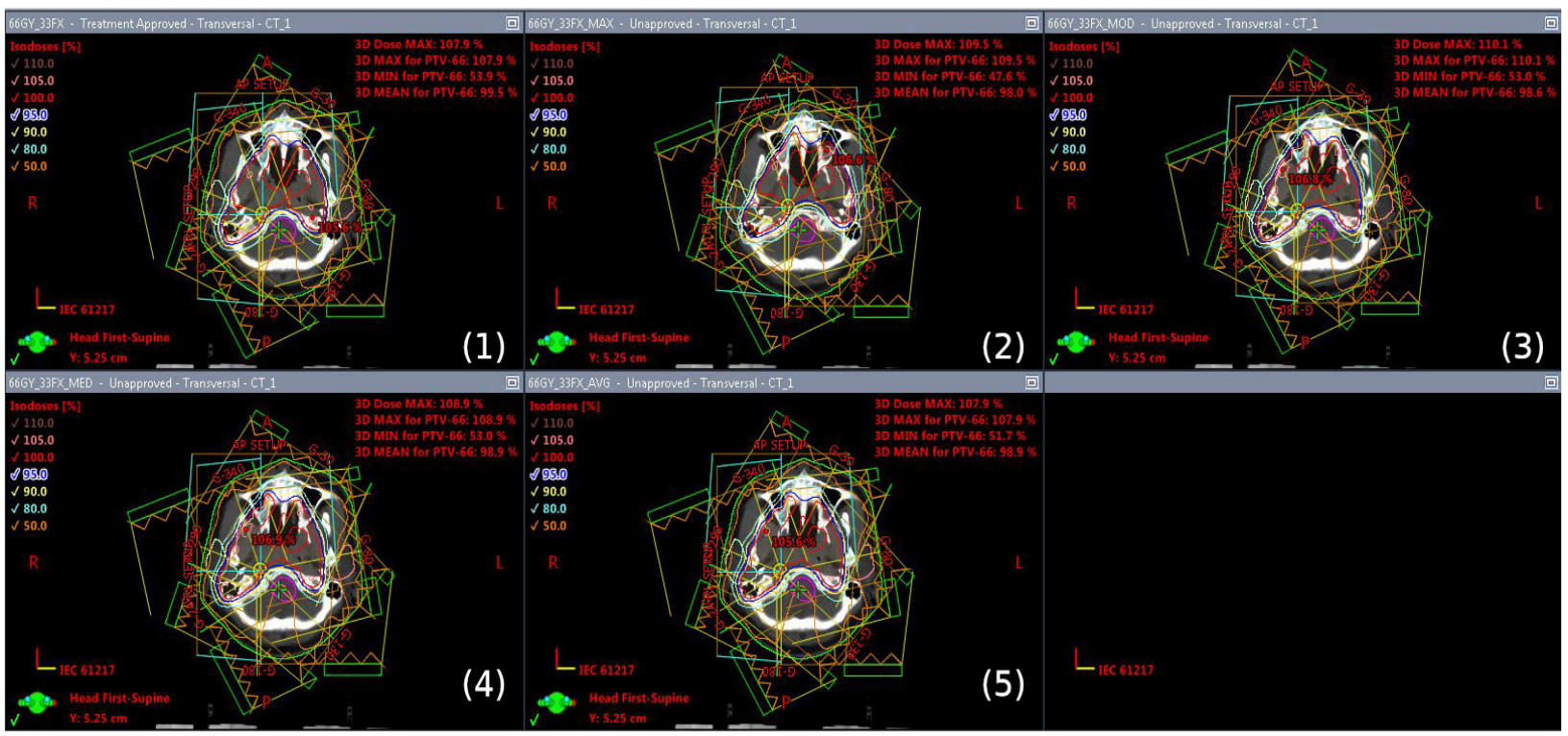

Figure 1. The Dose Distribution of Base Plan (1) and All Other Compared Plans of Maximum (2), Mode (3), Median (4) and Mean (5) Shift Plans Respectively 
Table 1. PTV Parameters for Base Plan and Shift Plans

\begin{tabular}{lccccccccc}
\hline Parameters & Base Plan & Maximum & Mode & Median & Mean & $\begin{array}{c}\text { P value } \\
\text { (Max vs }\end{array}$ & $\begin{array}{c}\text { P value } \\
\text { (Mode vs } \\
\text { Base) }\end{array}$ & $\begin{array}{c}\text { P value } \\
\text { (Median } \\
\text { vs Base) }\end{array}$ & $\begin{array}{c}\text { P value } \\
\text { (Mean } \\
\text { vs Base) }\end{array}$ \\
\hline Mean dose & $62.092 \pm 3.900$ & $61.196 \pm 4.140$ & $61.639 \pm 3.960$ & $61.723 \pm 4.050$ & $61.952 \pm 3.900$ & 0.002 & 0.012 & 0.021 & 0.052 \\
$\mathrm{D}_{2 \%}$ & $65.062 \pm 3.900$ & $65.982 \pm 4.120$ & $65.244 \pm 3.930$ & $65.262 \pm 3.890$ & $65.139 \pm 3.900$ & 0.028 & 0.057 & 0.059 & 0.148 \\
$\mathrm{D}_{98 \%}$ & $55.068 \pm 3.790$ & $47.784 \pm 7.010$ & $52.846 \pm 4.200$ & $53.452 \pm 4.570$ & $54.259 \pm 3.900$ & 0.004 & 0.004 & 0.009 & 0.01 \\
$\mathrm{D}_{50 \%}$ & $62.634 \pm 3.900$ & $62.294 \pm 4.000$ & $62.465 \pm 3.910$ & $62.634 \pm 3.930$ & $62.541 \pm 3.900$ & 0.001 & 0.073 & 1 & 0.09 \\
$\mathrm{D}_{80 \%}$ & $60.723 \pm 3.960$ & $59.443 \pm 4.640$ & $60.057 \pm 4.070$ & $59.560 \pm 3.890$ & $60.542 \pm 4.000$ & 0.002 & 0.017 & 0.091 & 0.078 \\
$\mathrm{Cl}_{95 \%}$ & $1.268 \pm 0.270$ & $1.203 \pm 0.260$ & $1.168 \pm 0.170$ & $1.169 \pm 0.170$ & $1.186 \pm 0.190$ & 0.516 & 0.246 & 0.247 & 0.354 \\
$\mathrm{HI}_{95 \%}$ & $0.160 \pm 0.020$ & $0.294 \pm 0.110$ & $0.199 \pm 0.036$ & $0.189 \pm 0.036$ & $0.174 \pm 0.020$ & 0.004 & 0.005 & 0.006 & 0.008 \\
$\mathrm{CN}_{95 \%}$ & $0.691 \pm 0.120$ & $0.599 \pm 0.100$ & $0.674 \pm 0.098$ & $0.697 \pm 0.103$ & $0.696 \pm 0.100$ & 0.04 & 0.504 & 0.86 & 0.891 \\
$\mathrm{COVI}$ & $0.920 \pm 0.040$ & $0.837 \pm 0.050$ & $0.879 \pm 0.050$ & $0.894 \pm 0.045$ & $0.900 \pm 0.047$ & 0 & 0.005 & 0.002 & 0.013 \\
$\mathrm{DGI}$ & $0.292 \pm 0.120$ & $0.271 \pm 0.070$ & $0.267 \pm 0.080$ & $0.267 \pm 0.078$ & $0.268 \pm 0.070$ & 0.43 & 0.31 & 0.303 & 0.332 \\
UDI & $98.904 \pm 68.670$ & $123.631 \pm 107.070$ & $99.559 \pm 75.990$ & $95.323 \pm 74.890$ & $96.500 \pm 73.960$ & 0.269 & 0.959 & 0.795 & 0.87 \\
\hline
\end{tabular}

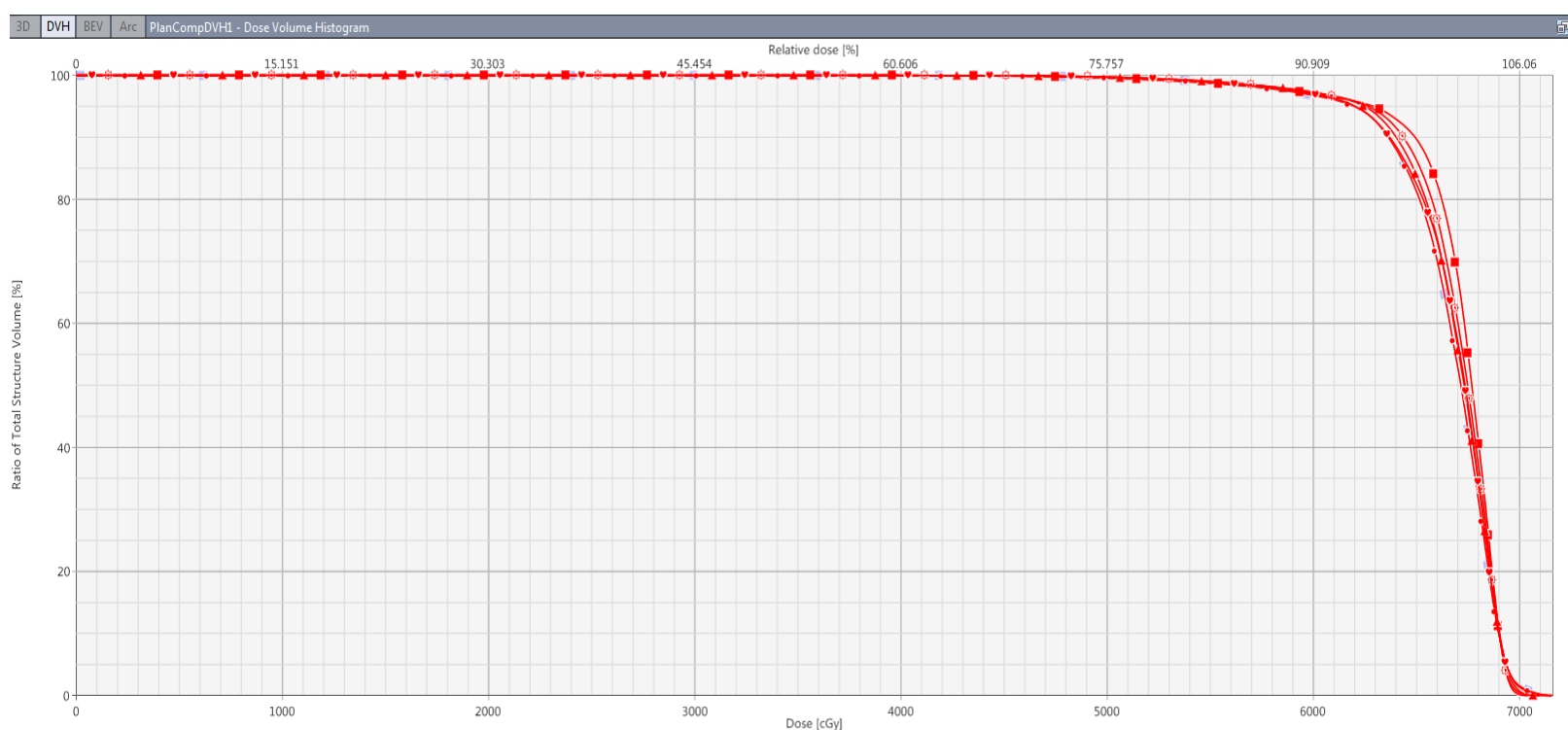

Figure 2. The DVH Comparison of PTV of a Patient for All the Plans

mode, median and mean shifts analyzed and noted with image guidance. The mean dose of PTV was [62.092 \pm 3.9 ] with the base plan which is reduced to a minimum of $[61.196 \pm 4.14]$ in maximum shift plan and the values are [61.639 \pm 3.96$],[61.723 \pm 4.05]$ and $[61.723 \pm 4.05]$ for mode, median and mean shift plans with a significant $\mathrm{P}$ value of $0.002,0.012,0.021,0.052$ for maximum, mode, median and mean shift plans respectively. The Conformity Index [CI95\%] is [1.268 \pm 0.27$]$ with the base plan which is changed to [1.203 \pm 0.26$],[1.168 \pm 0.17],[1.169 \pm 0.17]$ and [1.186 \pm 0.19$]$ for maximum, mode, median and mean shift plans. The Homogeneity Index [HI95\%] value of $[0.16 \pm 0.024]$ with the base plan is increased to a maximum value of $[0.294 \pm 0.109]$ with the maximum shift plan with the $\mathrm{P}$ value 0.004 and similarly higher when compared to the base plan for mode, median and mean shift plans. Likewise, the COVI value is better with a base plan as [0.92 \pm 0.04$]$ and it reduced to a minimum of $[0.837 \pm 0.05]$ with the maximum shift plan and similarly the lower for other shift plans with their significant $\mathrm{P}$ values $0.0004,0.005,0.002,0.013$ for maximum, mode, median and mean shift plans respectively. The Conformity Number [CN95\% ] value is [0.691 \pm 0.124$]$ with the base plan which reduced to a minimum of [0.599 \pm 0.095$]$ with the maximum shift plan. The DGI values obtained was higher with the base plan $[0.292 \pm 0.116]$ than maximum,

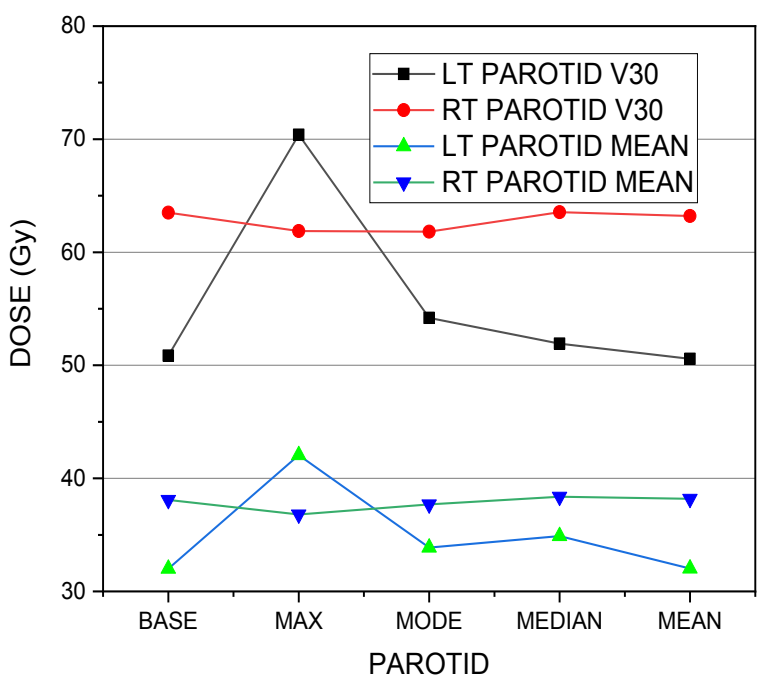

Figure 3. The Graphical Comparison of $\mathrm{V}_{30}$ and Mean Dose Values of Both Left Parotid and Right Parotid 
Table 2. OARs Parameters for Base Plan and Shift Plans

\begin{tabular}{|c|c|c|c|c|c|c|c|c|c|c|}
\hline \multirow[t]{2}{*}{ Parameters } & & \multirow[t]{2}{*}{ Base plan } & \multirow[t]{2}{*}{ Maximum } & \multirow[t]{2}{*}{ Mode } & \multirow[t]{2}{*}{ Median } & \multirow[t]{2}{*}{ Mean } & \multicolumn{4}{|c|}{ P Value } \\
\hline & & & & & & & Maximum & Mode & Median & Mean \\
\hline \multirow[t]{2}{*}{ Spinal cord } & $\mathrm{D}_{1 \%}$ & $37.67 \pm 2.20$ & $40.59 \pm 4.60$ & $38.66 \pm 4.20$ & $38.34 \pm 2.90$ & $40.51 \pm 6.50$ & 0.06 & 0.25 & 0.14 & 0.26 \\
\hline & $\mathrm{D}_{\max }$ & $40.77 \pm 2.10$ & $44.74 \pm 5.90$ & $42.77 \pm 4.90$ & $41.62 \pm 2.20$ & $41.21 \pm 1.70$ & 0.04 & 0.12 & 0.04 & 0.06 \\
\hline \multirow[t]{2}{*}{ Lt parotid } & Mean dose & $32.01 \pm 7.10$ & $42.06 \pm 6.90$ & $33.87 \pm 11.10$ & $34.90 \pm 13.40$ & $32.02 \pm 6.90$ & 0.00 & 0.26 & 0.26 & 0.99 \\
\hline & $\mathrm{V}_{30}$ & $50.86 \pm 16.80$ & $70.40 \pm 15.40$ & $54.20 \pm 23.40$ & $51.91 \pm 19.30$ & $50.57 \pm 16.70$ & 0.00 & 0.33 & 0.71 & 0.82 \\
\hline \multirow[t]{2}{*}{ Rt parotid } & Mean dose & $38.09 \pm 11.30$ & $36.81 \pm 11.30$ & $37.70 \pm 11.40$ & $38.38 \pm 12.80$ & $38.19 \div 12.10$ & 0.22 & 0.66 & 0.8 & 0.91 \\
\hline & $\mathrm{V}_{30}$ & $63.51 \pm 24.20$ & $61.88 \pm 26.30$ & $61.82 \pm 26.20$ & $63.54 \pm 26.90$ & $63.20 \pm 25.80$ & 0.48 & 0.44 & 0.99 & 0.86 \\
\hline \multirow[t]{2}{*}{ Larynx } & Mean dose & $42.12 \pm 12.00$ & $41.24 \pm 4.90$ & $39.74 \pm 7.50$ & $39.76 \pm 7.40$ & $41.5 \pm 10.70$ & 0.83 & 0.46 & 0.46 & 0.48 \\
\hline & $\mathrm{V}_{40}$ & $51.21 \pm 38.00$ & $53.84 \pm 23.80$ & $48.30 \pm 31.90$ & $48.39 \pm 31.60$ & $49.80 \pm 37.40$ & 0.81 & 0.52 & 0.53 & 0.05 \\
\hline Lt eye & Mean dose & $2.35 \pm 1.60$ & $3.34 \pm 4.20$ & $3.01 \pm 4.30$ & $2.26 \pm 2.10$ & $2.08 \pm 1.60$ & 0.33 & 0.53 & 0.75 & 0.19 \\
\hline Rt eye & Mean dose & $2.20 \pm 1.20$ & $3.24 \pm 3.20$ & $2.61 \pm 2.90$ & $2.16 \pm 1.60$ & $2.02 \pm 1.20$ & 0.18 & 0.55 & 0.89 & 0.22 \\
\hline Lt optic nerve & $\mathrm{D}_{1 \%}$ & $3.15 \pm 2.50$ & $8.55 \pm 14.20$ & $6.93 \pm 13.90$ & $2.33 \pm 0.90$ & $3.02 \pm 2.40$ & 0.21 & 0.36 & 0.29 & 0.28 \\
\hline Rt optic nerve & $\mathrm{D}_{1 \%}$ & $3.62 \pm 3.40$ & $10.15 \pm 16.04$ & $5.80 \pm 9.80$ & $3.77 \pm 3.90$ & $3.29 \pm 2.70$ & 0.16 & 0.44 & 0.85 & 0.32 \\
\hline Brainstem & Maximum dose & $40.44 \pm 8.82$ & $41.33 \pm 10.02$ & $38.53 \pm 11.75$ & $39.08 \pm 10.02$ & $39.54 \pm 9.78$ & 0.58 & 0.24 & 0.29 & 0.44 \\
\hline Mandible & Maximum dose & $62.94 \pm 4.00$ & $65.30 \pm 4.90$ & $63.96 \pm 4.40$ & $63.98 \pm 4.50$ & $63.31 \pm 4.30$ & 0.05 & 0.08 & 0.08 & 0.28 \\
\hline Lt cochlea & Mean dose & $14.11 \pm 15.90$ & $25.04 \pm 22.40$ & $21.87 \pm 23.60$ & $19.89 \pm 20.50$ & $14.56 \pm 14.90$ & 0.13 & 0.29 & 0.42 & 0.80 \\
\hline Rt cochlea & Mean dose & $17.97 \pm 26.30$ & $27.44 \pm 26.70$ & $23.51 \pm 28.30$ & $23.07 \pm 27.50$ & $18.25 \pm 25.50$ & 0.20 & 0.45 & 0.49 & 0.87 \\
\hline \multirow[t]{3}{*}{ Healthy tissue } & $\mathrm{V}_{5}$ & $27.46 \pm 8.30$ & $26.64 \pm 8.20$ & $27.46 \pm 8.70$ & $27.53 \pm 8.70$ & $27.63 \pm 8.50$ & 0.00 & 0.99 & 0.36 & 0.13 \\
\hline & $\mathrm{V}_{30}$ & $11.25 \pm 3.40$ & $10.99 \pm 3.40$ & $12.84 \pm 6.90$ & $11.16 \pm 3.40$ & $11.22 \pm 3.40$ & 0.00 & 0.38 & 0.12 & 0.34 \\
\hline & Mean dose & $8.20 \pm 2.40$ & $8.13 \pm 2.30$ & $8.18 \pm 2.50$ & $8.18 \pm 2.50$ & $8.21 \pm 2.40$ & 0.04 & 0.12 & 0.04 & 0.06 \\
\hline
\end{tabular}

mode, median and mean plan values of $[0.271 \pm 0.065]$, $[0.267 \pm 0.078],[0.267 \pm 0.078]$ and $[0.268 \pm 0.075]$. The UDI values obtained with the base plan was [98.904 \pm 68.67$]$ which reached a maximum value of [123.631 107.07$]$ with maximum shift plan.

Among the dose constraints of OARs, the spinal cord Dmax was increased from the base plan value of [40.77 \pm 2.1$]$ to a maximum of [44.74 \pm 5.9$]$ with the significant $\mathrm{P}$ value 0.0394 and similarly showed higher values with mode, median and mean shift plans with their $\mathrm{P}$ values $0.117,0.041$ and 0.064 respectively. The D1\% of the spinal cord reached a maximum of [40.59 \pm 4.6$]$ with the maximum shift plan from the base plan value of [37.67 \pm 2.2$]$ with its $P$ value 0.0554 . The 1 t parotid mean dose and V30 values shows a huge variation from its base plan values of $[32.01 \pm 7.1]$ and $[50.86 \pm 16.8]$ to its maximum shift plan values of [42.06 \pm 6.9$]$ and $[70.4 \pm 15.4]$ with its $\mathrm{P}$ values $9.35 \times 10^{-6}$ and $4.57 \times 10^{-6}$ . There was no significant dose difference between base plan and other shift plans for the mean dose and $\mathrm{V} 30$ values of Rt parotid. The V40 value of the larynx increased to a value of [53.84 \pm 23.8$]$ with the maximum shift plan from its base plan value of $[51.21 \pm 38.0]$ with its $\mathrm{P}$ value, 0.8139 . For lt eye, the maximum variation is seen between the base plan value of [2.35 \pm 1.6$]$ and maximum shift plan value of [3.34 \pm 4.2$]$ with the $P$ value, 0.3262 . Similarly with the right eye, a maximum variation of maximum shift plan value [3.24 \pm 3.2$]$ from its base plan value $[2.195 \pm 1.2]$ with a $P$ value of 0.1770 is noted. The maximum plan and mode plan values of D $1 \%$ of left optic nerve showed a large difference from its base plan value of [3.15 \pm 2.5$]$ to $[8.55 \pm 14.2]$ in the maximum shift plan and $[6.93 \pm 13.9]$ in the mode shift plan. Similarly, with the right optic nerve, the maximum and mode shift plans with their values $[10.15 \pm 16.04]$ and [5.80 \pm 9.8$]$ showed much variation from its base plan value of [3.62 \pm 3.4$]$. In the case of the brainstem, the maximum shift plan value changed as [41.33 \pm 10.02$]$ from the base plan value of $[40.44 \pm 8.82]$. For mandible, the maximum dose value of the maximum shift plan varied to [65.3 \pm 4.9$]$ from the base plan value of [62.94 \pm 4.0$]$ with a significant $P$ value of 0.0497 whereas for mode, median and mean shift plans, the P values noted are $0.081,0.079$ and 0.282 respectively. For right cochlea, the base plan value is $[17.97 \pm 26.3]$ which changed in maximum, mode, median and mean shift plans as [27.44 \pm 26.7$],[23.51 \pm 28.3]$, $[23.07 \pm 27.5]$ and $[18.25 \pm 25.5]$ respectively. Similarly for lt cochlea, the base plan value is [14.11 \pm 15.9$]$ which changed to [25.04 \pm 22.4$],[21.87 \pm 23.6],[19.89 \pm 20.5]$, [14.56 \pm 14.9$]$ in maximum, mode, median and mean shift plans respectively. With the healthy tissue, there was a little variation in the V5, V30 and mean dose values of the base plan from other shift plan values except for the V30 value [12.84 \pm 6.9$]$ of the mode shift plan from its base plan value of $[11.25 \pm 3.4]$.

\section{Discussion}

Different planning studies emphasize the importance of image guidance in radiotherapy treatment for tumor coverage (Ariyaratne et al., 2016; deCrevoisier et al., 2018; Wertz et al., 2007). Ariyaratne et al., (2016) did a study in which they showed that how it enhanced target scope day by day online in contrast with week by week imaging along with the decrease in rectal measurements and the possibility of PTV edge lessening to $5 \mathrm{~mm}$ with satisfactory target scope (Verma et al., 2014). From our study results it is very clear that in all the plans created by taking the maximum, mode, median and mean of the shift values inferred from image guidance, all the 
dosimetric parameters of PTV values deviated from the actual base plan. The dosimetric parameters which showed the maximum variations were a mean dose of maximum shift plan with a significant $P$ value 0.002 , D2\% of maximum shift plan with a significant $P$ value 0.028 , the D98\% values of maximum and mode shift plans with the significant $\mathrm{P}$ values 0.004 each, the D50\% and D80\% values of the maximum shift plan with their significant $\mathrm{P}$ values 0.001 and 0.002 respectively.

The HI95\%, CN95\% and COVI values of the maximum shift plan showed significant $P$ values of 0.004 , 0.040 and 0.0004 respectively from their base plan values. In a study conducted by Landoni et al., (2006), the effect of organ motion and setup errors on prostate cancer treatment with IMRT was analysed and it was concluded that by combining the patient motion and setup errors accurately, the treatment will be more defined. In another study by Wang et al., (2008) on the dosimetric effect of translational and rotational errors on spinal metastases patients treated with image-guided stereotactic body radiotherapy, it was stated that a $2 \mathrm{~mm}$ error in translational patient positioning in any direction will cause a tumor coverage loss of $>5 \%$ and an increase in maximal dose to the organs at risk by $>25 \%$. The study emphasized the importance of keeping the patient setup translational errors to $\leq 1 \mathrm{~mm}$ and rotational errors to $\leq 2$ degrees for obtaining the best dosimetric results in spinal stereotactic treatments.

It is clear from Table 2 of OARs that the shift plans showed a vast variation with an increase in its dosimetric parameter values from the actual base plan and the maximum variations was seen among the maximum shift plan and was mainly seen with the critical organs like the spinal cord, lt Parotid, right and left cochlea, mandible, right and left optic nerve. In a study, it was reported that for the spinal cord the maximum radiation dose that can be applied is 45Gy (Matzinger et al., 2009). The unique feature of this study from other studies is that, all the possible effective modes of shift errors and its dosimetric impact on the patient was studied. The study emphasized the importance of making image guidance a daily practice in radiotherapy treatment which is not followed in many centers as their protocol since they have to compromise with their patient work load. Image guidance is a mandatory procedure on daily basis in radiotherapy treatment for the precise treatment delivery and optimum results. Any amount of minimum to a maximum error in the patient setup will lead to a major missing of target and more dose exposure to critical organs which is very evident from the dosimetric results of the present study. In contrast to the studies of Tondel et al., (2018) who didn't demonstrate any advantage over daily imaging compared to weekly imaging, this study results dosimetrically reported the advantage of using daily imaging in radiotherapy which has to be mandatorily implemented in all radiotherapy centres for quality treatment.

\section{Author Contribution Statement}

The authors confirm contribution to the paper as follows: Study conception and design: Hridya V T, data collection analysis and interpretation of results: Hridya $\mathrm{V}$
T and Aswathi Raj, draft manuscript preparation: Hridya V T. All authors reviewed the results and approved the final version of the manuscript.

\section{Acknowledgements}

\section{Ethical Standards}

These articles don't contain any studies on human participants or animals by any of the authors.

\section{Data Availability}

Research Data are not shared

\section{Conflict of Interest}

All authors declare no conflict of interest.

\section{References}

Ariyaratne H, Chesham H, Pettingell J, Alonzi R (2016). Image guided radiotherapy for prostate cancer with cone beam CT: dosimetric effects of imaging frequency and PTV margin. Radiother Oncol, 121, 103-8.

Ashamalla H, Rafla S, Parikh K, et al (2005). The contribution of integrated PET/CT to the evolving definition of treatment volumes in radiation treatment planning in lung cancer. Int $J$ Radiat Oncol Biol Phys, 63, 1016-23.

Baskar R, Lee KA, Yeo R, Yeoh KW (2012). Cancer and radiation therapy: current advances and future directions. Int J Med Sci, 9, 193.

Cheung KY (2006). Intensity modulated radiotherapy: advantages, limitations and future developments. Biomed Imaging Interv $J, 2$, e19.

Contesini M, Guberti M, Saccani R, et al (2017). Setup errors in patients with head-neck cancer [HNC] treated using the Intensity Modulated Radiation Therapy [IMRT] technique: How it influences the customized immobilisation systems, patient's pain and anxiety. Radiat Oncol, 12, 1-7.

Crevoisier R, Bayar MA, Pommier P, et al (2018). Daily versus weekly prostate cancer image guided radiation therapy: phase 3 multicenter randomized trial. Int J Radiat Oncol Biol Phys, 102, 1420-29.

Ding GX, Alaei P, Curran B, et al (2018). Image guidance doses delivered during radiotherapy: Quantification, management, and reduction: Report of the AAPM Therapy Physics Committee Task Group 180. Med Phys, 45, 84-99.

Garibaldi C, Jereczek-Fossa BA, Marvaso G, et al (2017). Recent advances in radiation oncology. E Cancer Med Sci, 11.

Hummel S, Simpson EL, Hemingway P, Stevenson MD, Rees A (2010). Intensity-modulated radiotherapy for the treatment of prostate cancer: a systematic review and economic evaluation. Health Technol Assess, 14, 1-108.

Jaffray DA (2012). Image-guided radiotherapy: from current concept to future perspectives. Nat Rev Clin Oncol, 9, 688.

Kearney M, Coffey M, Leong A, (2020). A review of Image Guided Radiation Therapy in head and neck cancer from 2009-201-Best Practice Recommendations for RTTs in the Clinic. Tech Innov Patient Support Radiat Oncol, 14, 43-50.

Landoni V, Saracino B, Marzi S, et al (2006). A study of the effect of setup errors and organ motion on prostate cancer treatment with IMRT. Int J Radiat Oncol Biol Phys, 65, 587-94.

Matzinger O, Gerber E, Bernstein Z, et al (2009). EORTCROG expert opinion: radiotherapy volume and treatment guidelines for neoadjuvant radiation of adenocarcinomas of the gastroesophageal junction and the stomach. Radiother Oncol, 92, 164-75. 
Metcalfe P, Liney GP, Holloway L, et al (2013). The potential for an enhanced role for MRI in radiation-therapy treatment planning. Technol Cancer Res Treat, 12, 429-46.

Mohandass P, Khanna D, Nishaanth B, et al (2020). Impact of three different matching methods on patient set-up error in $\mathrm{x}$-ray volumetric imaging for head and neck cancer. Rep Pract Oncol Radiother, 25, 906-12.

Murshed H, Liu HH, Liao Z, et al (2004). Dose and volume reduction for normal lung using intensity-modulated radiotherapy for advanced-stage non-small-cell lung cancer. Int J Radiat Oncol Biol Phys, 58, 1258-67.

Nabavizadeh N, Elliott DA, Chen Y, et al (2016). Image guided radiation therapy (IGRT) practice patterns and IGRT's impact on workflow and treatment planning: Results from a national survey of American Society for Radiation Oncology members. Int J Radiat Oncol Biol Phys, 94, 850-7.

Nahum, AE, Uzan J (2012). Radio biological optimization of external-beam radiotherapy. Comput Math Methods Med, 2012.

Tondel H, Lund JÅ, Lydersen S, et al (2018). Radiotherapy for prostate cancer-Does daily image guidance with tighter margins improves patient reported outcomes compared to weekly orthogonal verified irradiation? Results from a randomized controlled trial. Radiother Oncol, 126, 229-35.

Van't Riet A, Mak AC, Moerland MA, Elders LH, Van Der Zee W (1997). A conformation number to quantify the degree of conformality in brachytherapy and external beam irradiation: application to the prostate. Int J Radiat Oncol Biol Phys, 37, 731-6.

Verma S, Sarabhai P (2014). Image-guided radiotherapy for prostate tumor with cone beam scanner and dosimetric aspects with planning target volume. Transl Phys Res, 3, 25-32.

Wang H, Shiu A, Wang C, et al (2008). Dosimetric effect of translational and rotational errors for patients undergoing image-guided stereotactic body radiotherapy for spinal metastases. Int J Radiat Oncol Biol Phys, 71, 1261-71.

Wertz H, Lohr F, Dobler B, et al (2007). Dosimetric consequences of a translational isocenter correction based on image guidance for intensity modulated radiotherapy (IMRT)of the prostate. Phys Med Biol, 52, 5655.

Weygand J, Fuller CD, Ibbott GS, et al (2016). Spatial precision in magnetic resonance imaging-guided radiation therapy: the role of geometric distortion. Int J Radiat Oncol Biol Phys, 95, 1304-16.

\section{@() $(0 \otimes$}

This work is licensed under a Creative Commons AttributionNon Commercial 4.0 International License. 